\title{
Article \\ Macrophage C/EBP\& Drives Gemcitabine, but Not 5-FU or Paclitaxel, Resistance of Pancreatic Cancer Cells in a Deoxycytidine-Dependent Manner
}

\author{
C. Arnold Spek ${ }^{1, *(D)}$, Hella L. Aberson ${ }^{1}$ and JanWillem Duitman ${ }^{2}$ D \\ 1 Center for Experimental and Molecular Medicine, Amsterdam UMC, University of Amsterdam, \\ 1105 AZ Amsterdam, The Netherlands; h.l.aberson@amsterdamumc.nl \\ 2 Department of Respiratory Medicine, Amsterdam UMC, University of Amsterdam, \\ 1105 AZ Amsterdam, The Netherlands; j.w.duitman@amsterdamumc.nl \\ * Correspondence: c.a.spek@amsterdamumc.nl
}

check for updates

Citation: Spek, C.A.; Aberson, H.L.; Duitman, J. Macrophage C/EBPS Drives Gemcitabine, but Not 5-FU or Paclitaxel, Resistance of Pancreatic Cancer Cells in a

Deoxycytidine-Dependent Manner. Biomedicines 2022, 10, 219.

https://doi.org/10.3390/

biomedicines10020219

Academic Editor: Satoshi Wada

Received: 20 December 2021

Accepted: 19 January 2022

Published: 20 January 2022

Publisher's Note: MDPI stays neutral with regard to jurisdictional claims in published maps and institutional affiliations.

Copyright: (C) 2022 by the authors. Licensee MDPI, Basel, Switzerland. This article is an open access article distributed under the terms and conditions of the Creative Commons Attribution (CC BY) license (https:// creativecommons.org/licenses/by/ $4.0 /)$.

\begin{abstract}
Treatment of pancreatic ductal adenocarcinoma (PDAC), a dismal disease with poor survival rates, is hampered by the high prevalence of chemotherapy resistance. Resistance is accompanied by macrophage infiltration into the tumor microenvironment, and infiltrated macrophages are key players in chemotherapy resistance. In the current manuscript, we identify CCAAT/enhancerbinding protein delta $(\mathrm{C} / \mathrm{EBP} \delta)$ as an important transcription factor driving macrophage-dependent gemcitabine resistance. We show that conditioned medium obtained from wild type macrophages largely diminishes gemcitabine-induced cytotoxicity of PDAC cells, whereas conditioned medium obtained from $\mathrm{C} / \mathrm{EBP} \delta$-deficient macrophages only minimally affects gemcitabine-induced PDAC cell death. Subsequent analysis of RNA-Seq data identified the pyrimidine metabolism pathway amongst the most significant pathways down-regulated in C/EBP $\delta$-deficient macrophages and size filtration experiments indeed showed that the low molecular weight and free metabolite fraction most effectively induced gemcitabine resistance. In line with a role for pyrimidines, we next show that supplementing macrophage conditioned medium with deoxycytidine overruled the effect of macrophage conditioned media on gemcitabine resistance. Consistently, macrophage C/EBP $\delta$-dependent resistance is specific for gemcitabine and does not affect paclitaxel or 5-FU-induced cytotoxicity. Overall, we thus show that $\mathrm{C} / \mathrm{EBP} \delta$-dependent deoxycytidine biosynthesis in macrophages induces gemcitabine resistance of pancreatic cancer cells.
\end{abstract}

Keywords: CCAAT/enhancer-binding protein delta; CEBPD; gemcitabine; drug resistance; pancreatic cancer; PDAC

\section{Introduction}

Pancreatic adenocarcinoma (PDAC) is a devastating disease with one of the worst survival rates of all human cancers [1]. Despite improvements in the treatment of cancer in general, hardly any progress has been made in PDAC treatment and the average survival rate remains around 6-8 months. Five years after diagnosis, more than $90 \%$ of PDAC patients have died, while 10-year mortality rates approach $99 \%$ [2,3]. The dismal prognosis of PDAC patients is largely due to the fact that most patients present with locally advanced or metastatic disease and are consequently ineligible for curative resection [4]. Chemotherapy-based therapies therefore remain standard of care [5], but chemotherapeutic treatments only minimally affect PDAC progression [6,7], therefore improving the efficacy of chemotherapy in PDAC is of utmost importance.

Macrophages are specialized phagocytic cells of the innate immune system critically involved in host defense and tissue homeostasis [8]. In cancer biology, macrophages are traditionally considered anti-tumorigenic, as seminal papers showed that macrophages may kill tumor cells by secreting cytotoxic molecules, such as TNF- $\alpha$, IL-12, nitric oxide 
(NO) and reactive oxygen species (ROS) $[9,10]$. Once recruited to the tumor, however, macrophages are reprogrammed to prevent killing of cancer cells. Such reprogrammed M2 or tumor associated macrophages (TAMs) not only show limited cytotoxicity towards cancer cells but actually become pro-tumorigenic and potentiate tumor growth [11-13]. Indeed, TAMs induce epithelial-to-mesenchymal transition (EMT) [14-16] and limiting macrophage infiltration decreases the number of metastatic lesions [17]. In addition, TAM numbers are associated with therapy resistance in PDAC $[18,19]$, and pharmacological depletion of TAMs enhanced the therapeutic response to gemcitabine in tumor-bearing KPC mice [20].

CCAAT/enhancer-binding protein delta (C/EBP $\delta)$, also known as nuclear factor interleukin (IL)-6 $\beta$, is a member of the C/EBP family of transcription factors [21]. It modulates many biological processes involved in cancer biology and C/EBP $\delta$ acts as tumor suppressor in multiple tumor types [22-25]. In PDAC, C/EBP $\delta$ is down-regulated in cancer cells, and reduced C/EBP $\delta$ expression correlates with poor prognosis [26]. As opposed to low

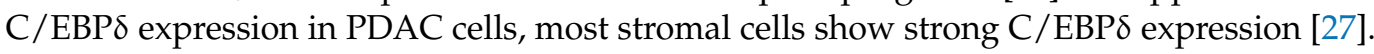
In particular, the expression of C/EBP $\delta$ in macrophages seems interesting, as C/EBP $\delta$ is well-known to regulate macrophage-dependent chemokine and cytokine expression [28], whereas C/EBPS also may affect macrophage polarization [29]. Considering the importance of cytokine production and macrophage polarization in drug resistance [30], we hypothesized that macrophage $\mathrm{C} / \mathrm{EBP} \delta$ would have an impact on drug resistance of PDAC cells. By using C/EBP $\delta$-deficient macrophages, we show that macrophage C/EBP $\delta$ drives gemcitabine, but not 5-FU or paclitaxel, resistance in a deoxycytidine-dependent manner.

\section{Materials and Methods}

\subsection{Cell Culture}

PANC-1, MIA PaCa-2 and BxPc3 PDAC cells (ATCC, Manassas, VA, USA) were cultured in DMEM (Lonza, Basel, Switzerland), whereas wild type and C/EBPS knock out RAW264.7 macrophages, generated and characterized as described previously [31], were cultured in IMDM medium (Gibco, Thermo Fischer Scientific, Waltham, MA, USA). All media were supplemented with $10 \%$ fetal calf serum (FCS), $2 \mathrm{mM}$ L-glutamine, 100 units $/ \mathrm{mL}$ penicillin, and $500 \mu \mathrm{g} / \mathrm{mL}$ streptomycin (all Lonza, Basel, Switzerland) according to routine cell culture procedures. Cells were maintained in a humidified incubator at $37^{\circ} \mathrm{C}$ and $5 \%$ CO2. All PDAC cell lines were authenticated by STR profiling (Promega PowerPlex, Leiden, Netherlands), and tested for mycoplasma by PCR monthly.

\subsection{Conditioned Medium Collection and PDAC Cytotoxicity Assays}

Wild type and C/EBP $\delta$ knock out RAW264.7 macrophages were grown to $70 \%$ confluence in a T75 flask, after which the growth medium was refreshed. After $24 \mathrm{~h}$, medium was collected, sterilized through a $0.2 \mu \mathrm{m}$ filter and stored at $-20^{\circ} \mathrm{C}$. PDAC cells, seeded in $100 \mu \mathrm{L}$ complete medium in 96-well plates were, upon adherence of the cells, incubated in conditioned medium (diluted 1:1 in complete DMEM) supplemented with gemcitabine, 5-FU or paclitaxel. Based on IC50 analysis (Figures S1 and S3), PANC-1 cells were treated with $20 \mathrm{nM}$ gemcitabine, $5 \mathrm{nM}$ paclitaxel or $4 \mu \mathrm{M}$ 5-FU, MIA PaCa-2 cells were treated with $15 \mathrm{nM}$ gemcitabine, $20 \mathrm{nM}$ paclitaxel or $2 \mu \mathrm{M}$ 5-FU, whereas BxPc3 cells were treated with $10 \mathrm{nM}$ gemcitabine, $4 \mathrm{nM}$ paclitaxel or $4 \mu \mathrm{M}$ 5-FU. After $96 \mathrm{~h}$, drug-induced cytotoxicity was assessed essentially as described before [32]. In detail, PDAC cells were washed and incubated with crystal violet ( $3 \%$ formaldehyde, $0.5 \%$ crystal violet in $\mathrm{H}_{2} \mathrm{O}$ ) at room temperature. After $20 \mathrm{~min}$, the crystal violet solution was removed, cells were washed 3 times with tap water, and $75 \mu \mathrm{L} /$ well DMSO was added to solubilize the formed crystals. After 20 min of incubation on a plate shaker at room temperature, the absorbance was measured at $600 \mathrm{~nm}$ on a Synergy HT plate reader (BioTek Instruments, Winooski, VT, USA). 


\subsection{Size Separation of Proteins in Conditioned Media}

RAW264.7 conditioned media, collected as described above, were separated using 10 kD Molecular Weight Cut Off ultra-centrifugal filters (Amicon-Ultra, Millipore, Ireland). The concentrated $>10 \mathrm{kD}$ fraction was resuspended in IMDM to the original volume before storage at $4{ }^{\circ} \mathrm{C}$, whereas the flow-through fraction $(<10 \mathrm{kD})$ was stored at $4{ }^{\circ} \mathrm{C}$ until used in experiments.

\subsection{RT-qPCR and RNA-Seq}

RNA was extracted form TriReagent lysed cells according to routine procedures. Eluted RNA was analyzed spectrophotometrically using a NanoDrop 2000 (Thermo Fisher Scientific, Waltham, MA, USA). All samples were treated with RQ1 RNAse-Free DNAse (Promega Benelux BV) and reverse-transcribed into cDNA using M-MLV Reverse Transcriptase (Promega Benelux BV, Leiden, The Netherlands), random hexamers (Fisher scientific) and $10 \mathrm{mM}$ dNTPs (Fermentas, Fisher scientific, Landsmeer, The Netherlands). The SensiFAST $^{\mathrm{TM}}$ SYBR ${ }^{\circledR}$ No-ROX Kit (GC biotech, Waddinxveen, The Netherlands) was used to perform real-time quantitative RT-PCR on a LightCycler ${ }^{\circledR} 480$ Instrument II (Roche Molecular Systems, Inc., Almere, The Netherlands). Expression levels were normalized to the expression of the reference genes TBP, B2M and UBC using the primers listed in Supplementary Table S1. RNA-Seq results and the corresponding experimental design were described previously [31] and represent transcriptional differences between wild type and C/EBP $\delta$-deficient RAW264.7 cells. The complete sequence libraries are publicly available through the National Center for Biotechnology Information gene expression omnibus under the following accession number: GSE173552. Differential gene expression was analyzed using the R2 microarray analysis and visualization platform (R2: Genomics Analysis and Visualization Platform. Available online: http:/ / r2.amc.nl; last accessed 12 January 2022) with a false discovery adjusted $p$-value less than 0.01 . To analyze the function of the differentially expressed genes, Kyoto Encyclopedia of Genes and Genomes (KEGG) pathway enrichment analyses were conducted with a $p$-value cutoff off less than 0.005 .

\subsection{Statistical Analysis}

All data are expressed as means \pm SEM. Differences between multiple groups were analyzed by one-way ANOVA with Bonferroni correction for multiple testing, whereas t-tests were used for comparisons between 2 groups. Analyses were performed using GraphPad Prism version 8 (GraphPad Software, Inc., La Jolla, CA, USA). Statistically significant differences were considered with a $p$-value less than 0.05 .

\section{Results}

\subsection{Macrophage C/EBPS Drives Gemcitabine Resistance of Pancreatic Cancer Cells}

To investigate the importance of $\mathrm{C} / \mathrm{EBP} \delta$ in macrophage-dependent resistance to gemcitabine, we employed wild type and C/EBPS-deficient RAW264.7 macrophages. Conditioned medium of these cells was compared to control medium in gemcitabine-induced pancreatic cancer cell cytotoxicity assays (Figure 1A). As shown in Figure 1B-D, conditioned medium obtained from wild type macrophages induced gemcitabine resistance in MIA PaCa-2 (Figure 1B), BxPc3 (Figure 1C) and PANC-1 (Figure 1D) cells. Of note, conditioned medium obtained from $\mathrm{C} / \mathrm{EBP} \delta$-deficient macrophages did not affect gemcitabine-induced cytotoxicity of any of the pancreatic cancer cells tested. To exclude a direct effect of conditioned medium on cell viability, MIA PaCa-2 cells were incubated in conditioned medium in the absence of gemcitabine, showing that conditioned medium indeed did not affect cell viability by itself (Figure S2). 
A
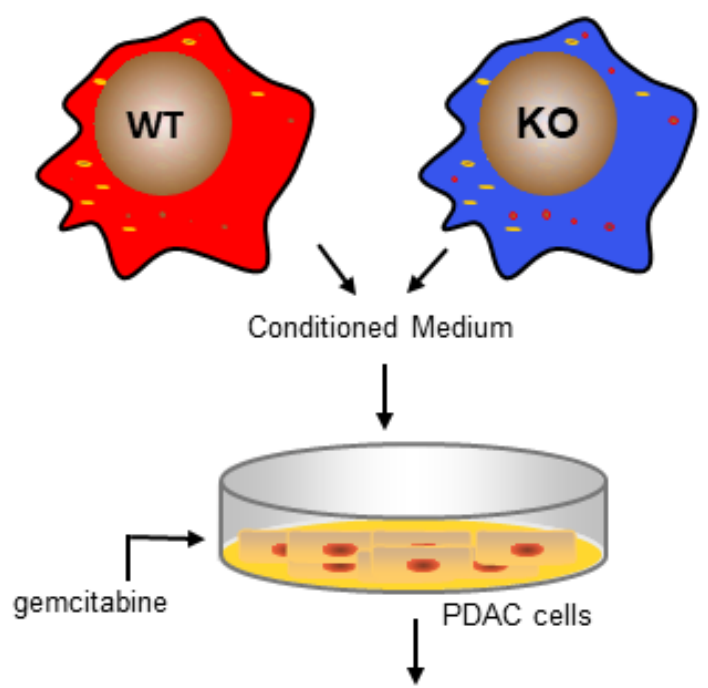

Viability

B

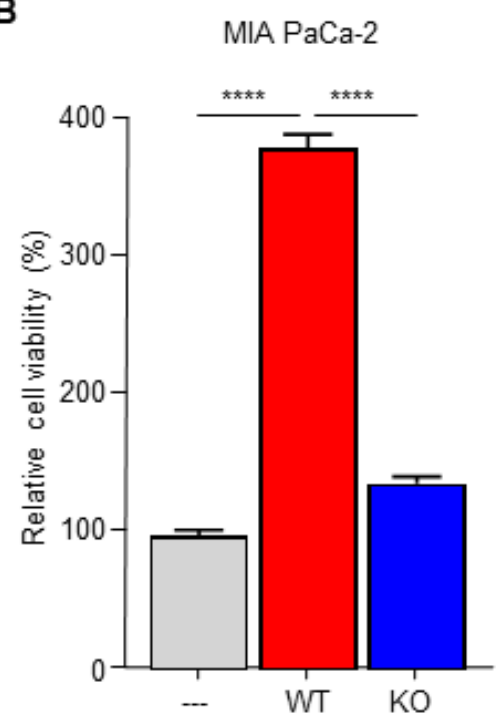

C

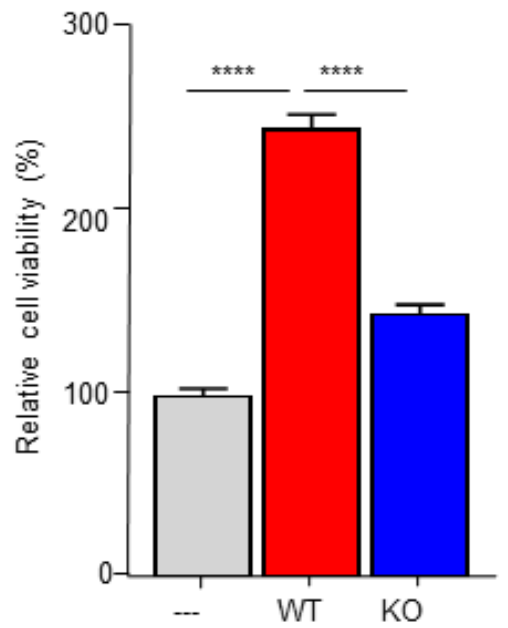

D

PANC-1

Figure 1. Macrophage C/EBP $\delta$ drives gemcitabine resistance of pancreatic cancer cells. (A) Schematic overview of the experimental set up. (B-D) Viability of Mia PaCa-2 (B), BxPc3 (C) and PANC-1 (D) pancreatic cancer cells in control medium (-), conditioned medium obtained from wild type macrophages (WT) and conditioned medium obtained from C/EBP $\delta$-deficient macrophages (KO) in the presence of gemcitabine. Shown is the mean \pm SEM of two to three independent experiments performed in sixplo. ${ }^{* * * *} p<0.0001$.

\subsection{C/EBP $\delta$ Deficiency Inhibits Pyrimidine Syntheses in Macrophages}

Macrophage-secreted chemokines and growth factors are suggested to induce drug resistance [18], suggesting that macrophage C/EBP $\delta$ potentiates gemcitabine resistance by enhancing chemokine/cytokine secretion. Analysis of recently obtained RNA-Seq data to determine transcriptional changes induced by C/EBP $\delta$ deletion in macrophages (GSE173552 [31]) revealed, however, no apparent changes in the expression of chemokines/cytokines. KEGG pathway enrichment analyses of differentially expressed genes between wild type and C/EBP $\delta$-deficient macrophages, in fact, identified the pyrimidine metabolism pathway amongst the most significant pathways down-regulated by C/EBP $\delta$ deficiency (Figure 2A). Detailed analysis of the pyrimidine metabolism pathway showed 
that 34 genes were significantly decreased in C/EBP $\delta$-deficient macrophages (Figure 2B) of which the 9 most down-regulated genes were confirmed by RT-PCR analysis (Figure 2C).

A

Ribosome biogenesis

RNA transport

Spliceosome

mRNA surveillance

RNA polymerase

Pyrimidine metabolism

Central carbon metabolism in cancer

Protein processing in ER

Proteasome

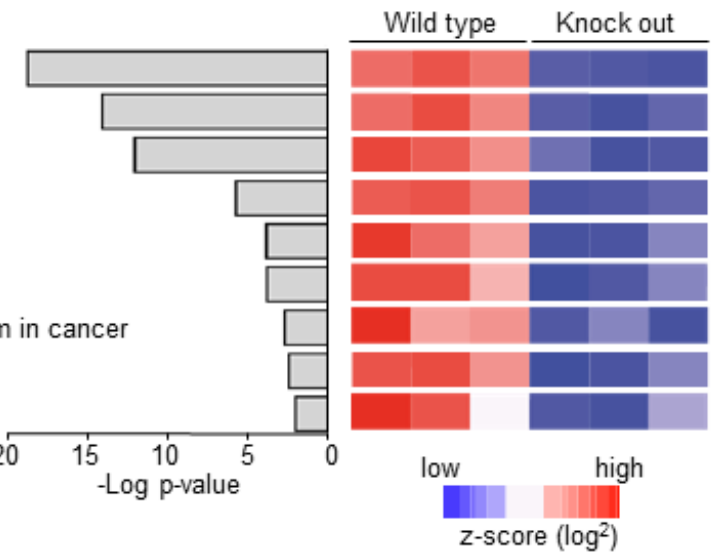

$B_{\text {low }}$
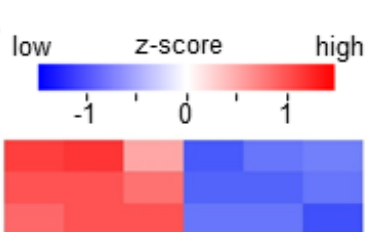

DPYS

RRM2

TWISTNB

ENTPD5

NME6

POLR1B

POLR3D

CTPS

UMPS

POLR2H

POLR1E

DCTD

POLR3F

CAD

POLR2I

UCK2

POLR1A

NME1

POLR3C

ENTPD6

RRM2B

ITPA

POLR3A

DUT

POLR2C

POLE3

TK1

POLR2F

NT5C2

POLR3B

POLR3E

UPP1

POLA2

PRIM1
C
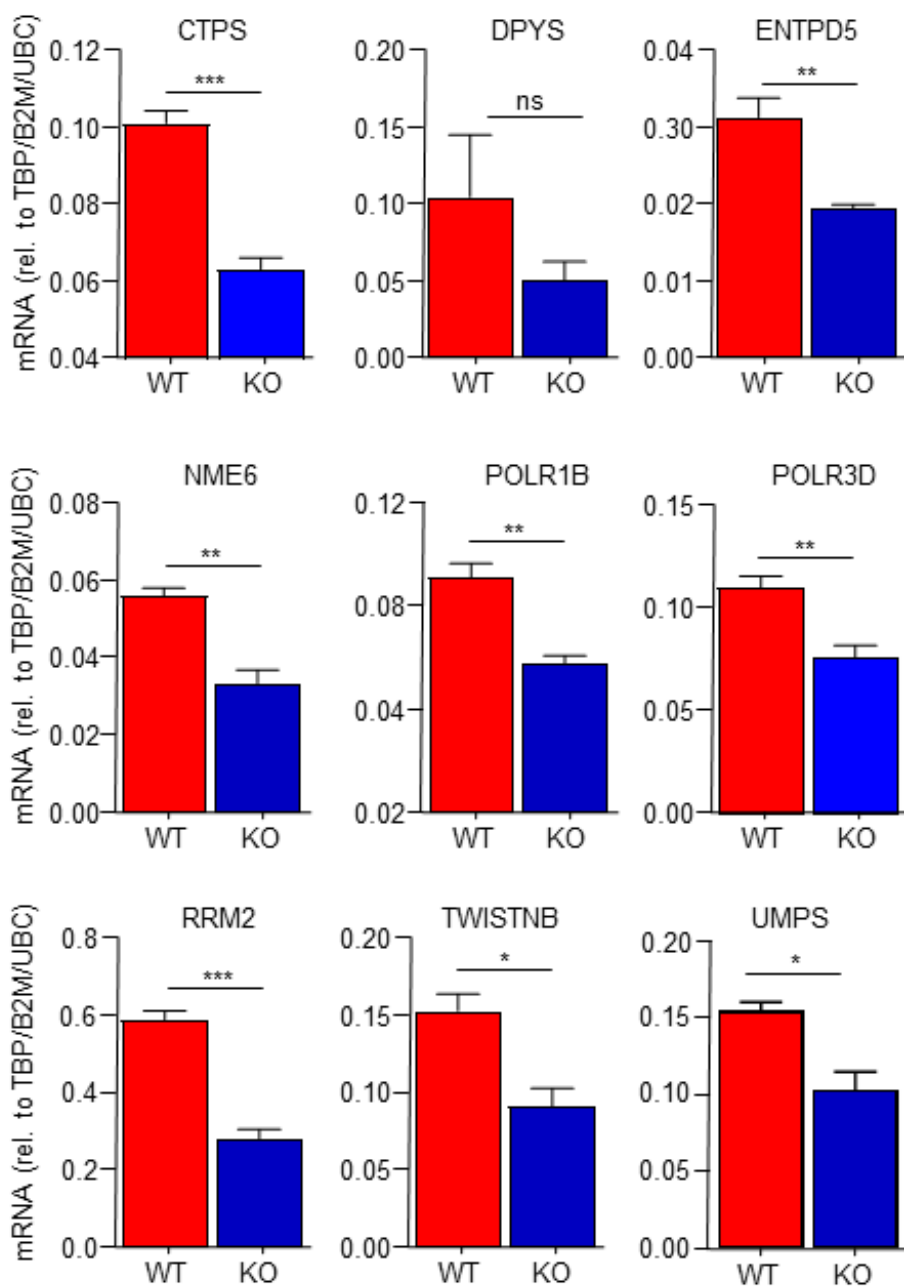

Figure 2. C/EBP $\delta$ deficiency inhibits pyrimidine syntheses. (A) The $p$-values and names of the most over-represented KEGG pathways, calculated on the basis of all the differentially expressed genes between wild type and C/EBP $\delta$-deficient macrophages. (B) Heatmap of all the differentially expressed genes from the pyrimidine metabolism pathway. (C) RT-PCR validation of the top differentially expressed genes of the pyrimidine metabolism pathway. ${ }^{*} p<0.05 ;{ }^{* *} p<0.01$; ${ }^{* *} p<0.005$; ns: not significant. 


\subsection{Macrophage C/EBPS Drives Gemcitabine Resistance in a Deoxycytidine-Dependent Manner}

The pyrimidine metabolism pathway has previously been shown to affect gemcitabine resistance [33,34]. Indeed, deoxycytidine directly competes with gemcitabine thereby inhibiting its uptake and activation, leading to diminished intracellular gemcitabine activity and reduced gemcitabine-induced cytotoxicity [33]. It is thus tempting to speculate that macrophage $\mathrm{C} / \mathrm{EBP} \delta$ drives gemcitabine resistance in a deoxycytidine-dependent manner. To prove or refute this hypothesis, we first subjected conditioned medium obtained from wild type macrophages to a size filtration spin column with a $10 \mathrm{kD}$ cutoff to separate proteins from free metabolites (Figure 3A). As shown in Figure 3B, the flow through the fraction containing free metabolites was almost as effective in inducing gemcitabine resistance as control, non-filtrated, conditioned medium. The fraction that retained on the filter (containing proteins but also residual metabolites) was clearly less effective in inducing gemcitabine resistance of pancreatic cancer cells. In line with these findings that suggest that the resistance-inducing factor is not a protein, heat denaturation of the conditioned medium $\left(15 \mathrm{~min} 100^{\circ} \mathrm{C}\right.$ ) prior to transfer to pancreatic cancer cells did not diminish gemcitabine resistance (Figure 3C). Based on a model in which C/EBP $\delta$ dependent deoxycytidine biosynthesis in macrophages induces gemcitabine resistance of pancreatic cancer cells, exogenous administration of deoxycytidine should overrule the effect of macrophage C/EBP $\delta$. As shown in Figure 3D-F, deoxycytidine co-treatment indeed limits gemcitabine-induced cytotoxicity to a similar extent in control, wild type and $\mathrm{C} / \mathrm{EBP} \delta$-deficient conditioned medium. Of note, supplementing control or C/EBP $\delta$ deficient conditioned medium with low dose deoxycytidine $(0.4 \mathrm{uM})$ already results in similar gemcitabine resistance as induced by wild type conditioned medium (compare the $0.4 \mathrm{uM}$ bars of panels $\mathrm{D}$ and $\mathrm{F}$ with the $0 \mathrm{uM}$ bar of panel E). Supplementing control or conditioned medium with the pyrimidine deoxyguanosine did not induce gemcitabine resistance (Figure 3G-I), in line with the notion that deoxyguanosine does not compete with gemcitabine uptake and activation.

A

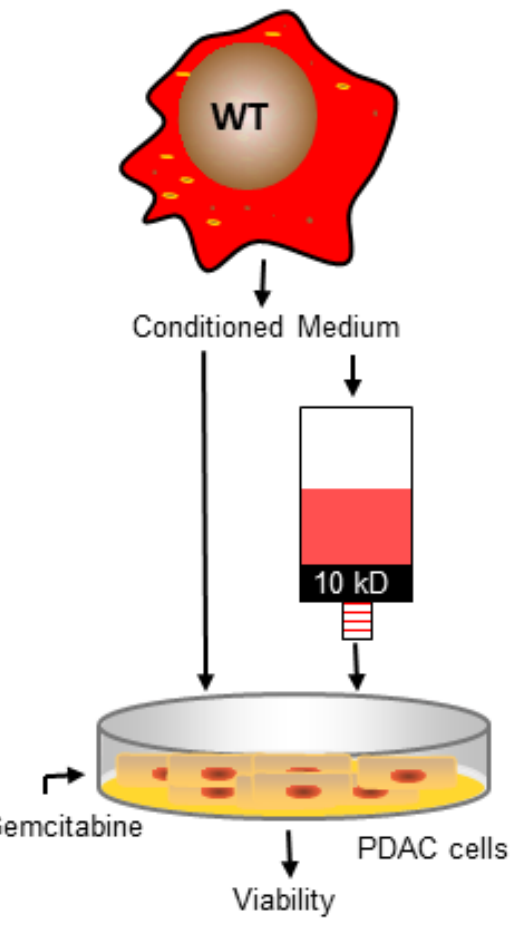

B

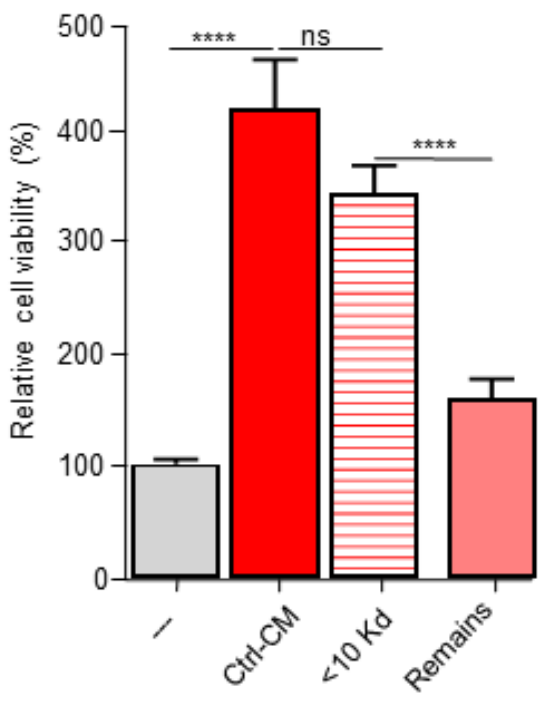

C

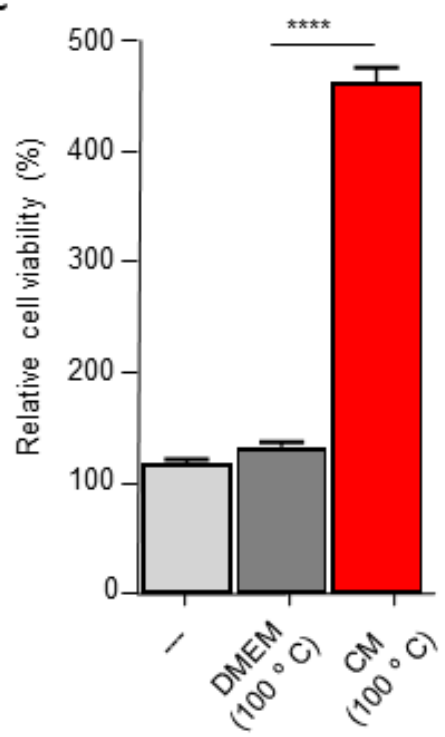

Figure 3. Cont. 


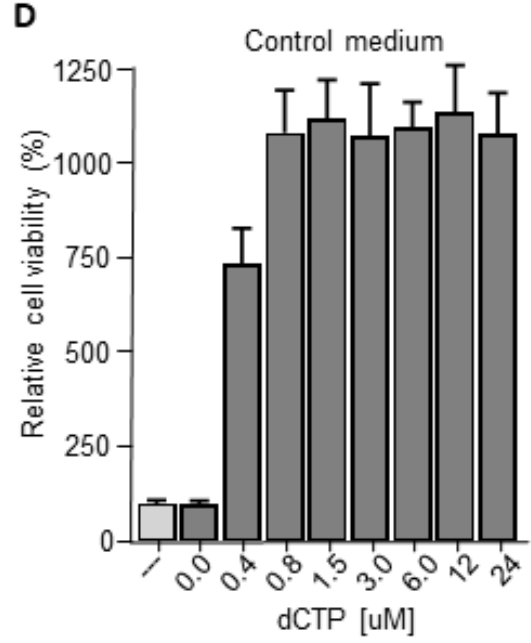

G

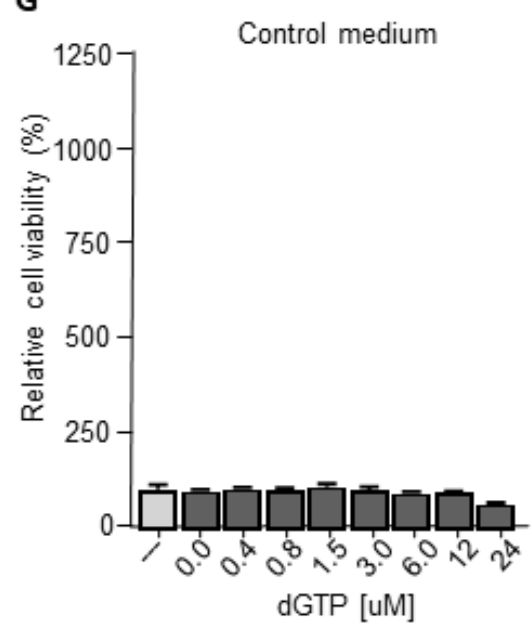

E

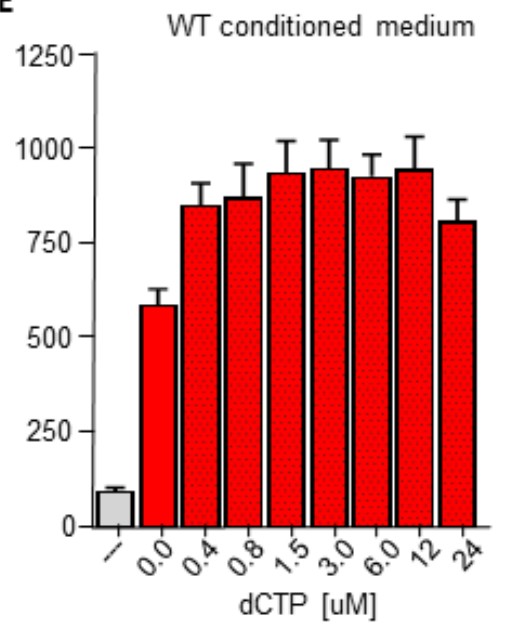

H

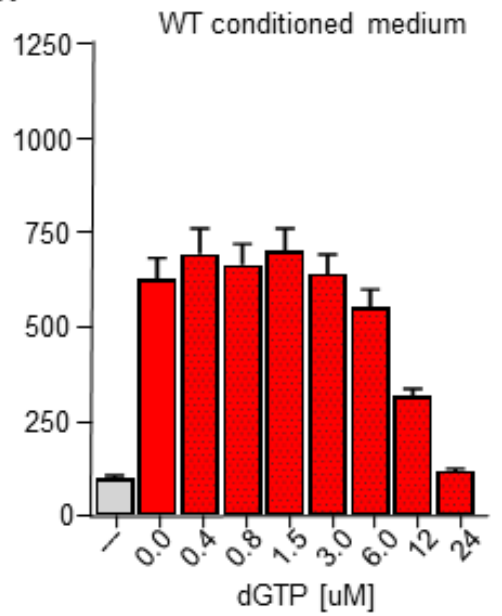

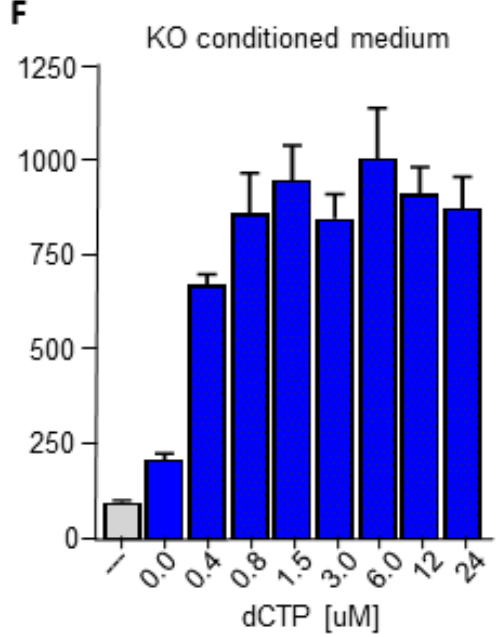

1

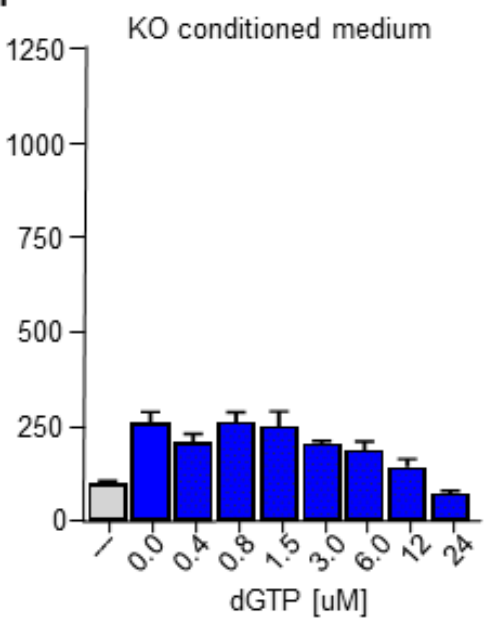

Figure 3. Macrophage C/EBP $\delta$ drives gemcitabine resistance in a deoxycytidine-dependent manner. (A) Schematic overview of the experimental set up. (B) Viability of Mia PaCa-2 pancreatic cancer cells in control medium (-), unfractionated conditioned medium obtained from wild type macrophages (Ctrl-CM), in the flow through fraction containing free metabolites $(<10 \mathrm{kD})$ and in the fraction that retained on the filter (remains) in the presence of gemcitabine. ${ }^{* * *} p<0.0001$; ns: not significant. (C) Viability of Mia PaCa-2 pancreatic cancer cells in control medium (-), boiled DMEM (DMEM $100{ }^{\circ} \mathrm{C}$ ) or boiled conditioned medium obtained from wild type macrophages $\left(\mathrm{CM} 100{ }^{\circ} \mathrm{C}\right)$ in the presence of gemcitabine. ${ }^{* * *} p<0.0001$. (D-F) Viability of Mia PaCa-2 pancreatic cancer cells in control medium (D) or conditioned medium obtained from wild type (E) or C/EBP $\delta$-deficient macrophages $(\mathbf{F})$ in the presence of gemcitabine and the indicated concentration of deoxycytidine (dCTP). (G-I) Viability of Mia PaCa-2 pancreatic cancer cells in control medium (G) or conditioned medium obtained from wild type $(\mathbf{H})$ or $\mathrm{C} / \mathrm{EBP} \delta$-deficient macrophages $(\mathbf{I})$ in the presence of gemcitabine and the indicated concentration of deoxycytidine (dCTP). Shown is the mean \pm SEM of experiments performed in sixplo. Note that the light grey bars in panels (D-I) (-) represent cell viability in control conditions (i.e., control medium supplemented with gemcitabine).

\subsection{Macrophage C/EBPS-Dependent Resistance of Pancreatic Cancer Cells Is Specific for Deoxycytidine Analogs}

The data above suggest that macrophage $\mathrm{C} / \mathrm{EBP} \delta$ induces gemcitabine resistance by potentiating deoxycytidine biosynthesis and secretion. For this hypothesis to be true, macrophage $\mathrm{C} / \mathrm{EBP} \delta$-induced resistance should be limited to gemcitabine. Consequently, we next assessed the effect of macrophage C/EBP $\delta$ on paclitaxel-, a cytotoxic agent routinely used in pancreatic cancer treatment that acts independent of DNA replication, and 5-FU-, a pyrimidine nucleoside-based cytotoxic agent with different transport and acti- 
vation properties as deoxycytidine [33], induced cell death (Figure 4A). In line with our hypothesis, conditioned medium obtained from both wild type and C/EBPS-deficient macrophages did not induce resistance to 5-FU or paclitaxel in MIA PaCa-2 (Figure 4B), PANC-1 (Figure 4C) or BxPc3 (Figure 4D) cells. Of note, conditioned medium did slightly enhance 5-FU- and paclitaxel-induced cytotoxicity in PANC1 and BxPc3 cells. Except for 5-FU-treated $\mathrm{BxPc} 3$ cells, this effect was similar for conditioned medium obtained from wild type and C/EBP $\delta$-deficient macrophages.

A
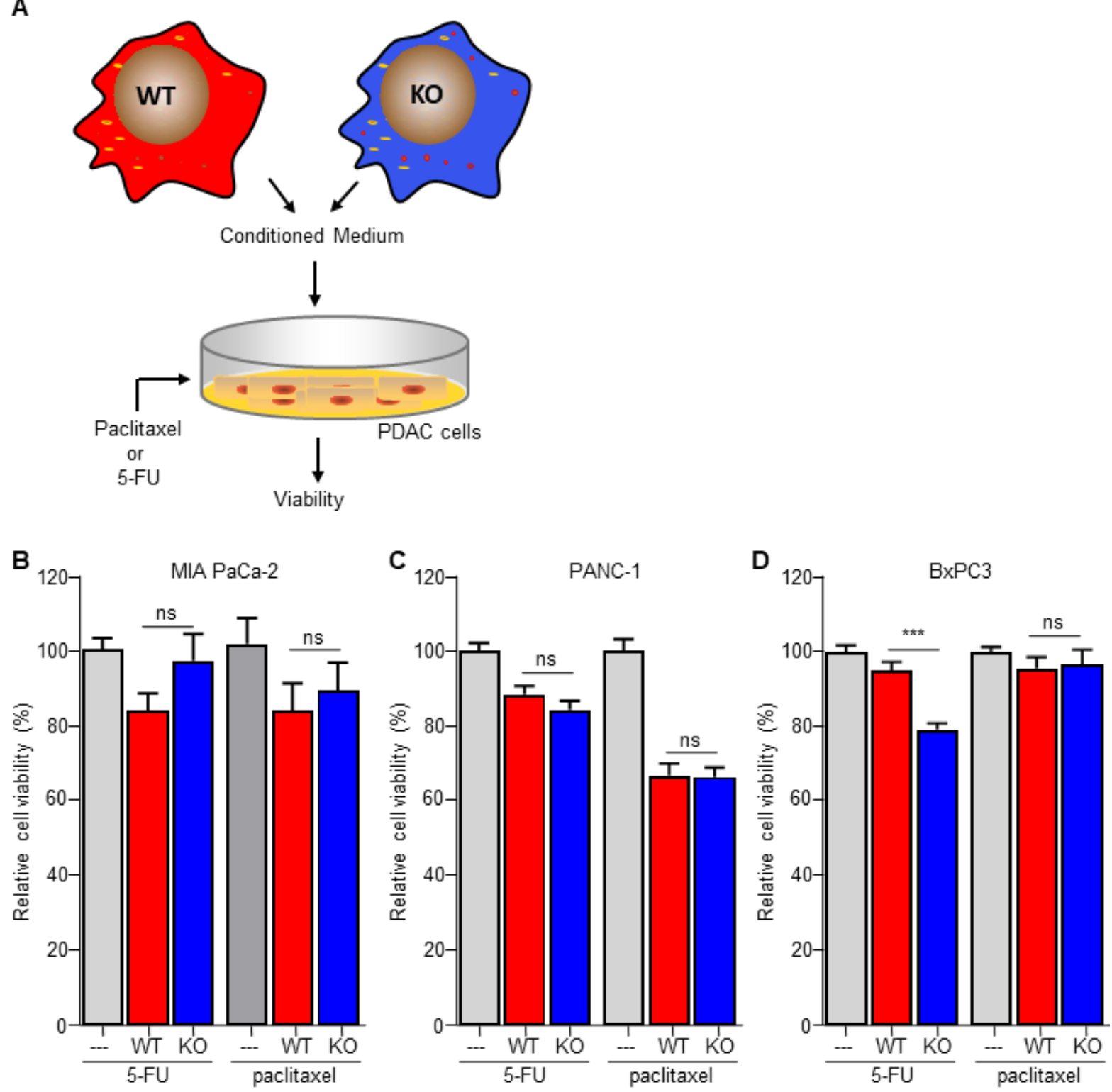

Figure 4. Macrophage C/EBP selectively induces resistance to gemcitabine. (A) Schematic overview of the experimental set up. (B-D) Viability of Mia PaCa-2 (B), BxPc3 (C) and PANC-1 (D) pancreatic cancer cells in control medium (-), conditioned medium obtained from wild type macrophages (WT) and conditioned medium obtained from C/EBP $\delta$-deficient macrophages $(\mathrm{KO})$ in the presence of 5-FU or paclitaxel. Shown is the mean \pm SEM of an experiment performed in sixplo. ${ }^{* * *} p<0.0001$; ns: not significant.

\section{Discussion}

In the current study, we aimed to elucidate the importance of macrophage C/EBP $\delta$ in drug resistance of pancreatic cancer cells. We confirm that macrophage-derived condi- 
tioned medium induces gemcitabine resistance of pancreatic cancer cells and show that conditioned medium obtained from $\mathrm{C} / \mathrm{EBP} \delta$-deficient macrophages no longer induces gemcitabine resistance. The fact that resistance, which occurs in most PDAC patients within weeks of treatment initiation, profoundly limits the efficacy of gemcitabine treatment [35], underscores the potential importance of our findings and suggests that C/EBP $\delta$ is a key factor underlying the poor prognosis of PDAC patients.

Single agent gemcitabine chemotherapy has been the backbone of PDAC treatment for many years [36-39], but combination therapy with folinic acid, fluorouracil, irinotecan and oxaliplatin (FOLFIRINOX) recently replaced gemcitabine as first-line treatment modality [40]. The increased clinical benefit of FOLFIRINOX comes, however, at the expense of increased toxicity [41]. In view of the substantial toxicity of FOLFIRINOX, understanding the underlying mechanisms by which PDAC patients become resistant to gemcitabine is not just of scientific importance. The identification of $\mathrm{C} / \mathrm{EBP} \delta$ as a driver of macrophagedependent gemcitabine resistance suggests that patients with low $\mathrm{C} / \mathrm{EBP} \delta$ expression in their macrophages may be particularly eligible for gemcitabine treatment. On the contrary, patients with high macrophage $\mathrm{C} / \mathrm{EBP} \delta$ levels should preferably not be treated with gemcitabine, as they are prone to be resistant and experience limited benefit of treatment. Future studies should prove or refute the validity of this tantalizing hypothesis.

Macrophage numbers in or around tumors are typically associated with poor prognosis and reduced overall survival [11-13]. In line with this, pharmacological macrophage depletion was recently shown to enhance the therapeutic response to gemcitabine in a preclinical murine PDAC model [20]. These data not only confirm the general concept that macrophages, despite their cytotoxic nature, exert a pro-tumorigenic role [42] but also suggest that macrophage depletion could be an attractive option to increase the clinical efficacy of gemcitabine. Of note, however, is that specific macrophage subsets may possess tumor-suppressive functions based upon which it has been suggested that macrophage reprogramming rather than depletion may be the most promising strategy to pursue $[20,43]$. We identify $\mathrm{C} / \mathrm{EBP} \delta$ as an attractive candidate to reprogram macrophages in the setting of gemcitabine therapy in PDAC patients. Our data suggest that combination therapy of gemcitabine and a C/EBP $\delta$ inhibitor will enhance gemcitabine efficacy and will improve treatment response in PDAC patients. In particular, patients with high C/EBP $\delta$ positive macrophage numbers are likely to benefit from combination therapy, but this hypothesis needs to be addressed in preclinical PDAC models before one could pursue to clinical studies. Although no C/EBPS inhibitor has yet been clinically approved, a recent study showed that two histone deacetylase (HDAC) inhibitors, suberoylanilide hydroxamic acid (SAHA) and trichostatin A (TSA), abolished endogenous C/EBP $\delta$ mRNA expression levels in THP-1 macrophages [44]. Whether these HDAC inhibitors do reduce C/EBP $\delta$ levels in tumor associated macrophages and subsequently potentiate gemcitabine efficacy in PDAC remains to be established in future experiments.

$\mathrm{C} / \mathrm{EBP} \delta$ plays a pleiotropic role in cancer biology and may either potentiate or inhibit cancer progression in a context-dependent manner. In PDAC, tumor cell C/EBP $\delta$ expression is lost and re-expression of $\mathrm{C} / \mathrm{EBP} \delta$ in PDAC cell lines slowed down proliferation and decreased the clonogenic capacity [26]. In contrast, however, primary tumor growth was not affected in C/EBP $\delta$-deficient mice but metastases were observed in numerous organs of tumor cell grafted wild type mice but not in $\mathrm{C} / \mathrm{EBP} \delta$-deficient mice [27]. Here, we extend the notion that $\mathrm{C} / \mathrm{EBP} \delta$ seems to exert opposing effects in tumor and non-tumor cells by showing that macrophage $\mathrm{C} / \mathrm{EBP} \delta$ limits the cytotoxicity of gemcitabine towards pancreatic cancer cells. Of note, a previous study suggested that macrophage C/EBP $\delta$ induced chemoresistance of breast cancer cells to both cisplatin and 5-FU [45]. The latter is especially interesting as we here show that wild type and C/EBP $\delta$-deficient macrophages both do not induce resistance of pancreatic cancer cells towards 5-FU, further underscoring the context-dependent role of C/EBP $\delta$ in tumor biology.

Gemcitabine resistance of pancreatic cancer cells is multifactorial [46] and may result from pancreatic cancer cell autonomous processes, for instance mitochondria-mediated 
apoptosis [47,48] or nucleoside transporter down-regulation [49], and from interactions between pancreatic cancer cells and stromal cells. Tumor associated macrophages may induce gemcitabine resistance of pancreatic cancer cells by several mechanisms (excellently reviewed by Yang and colleagues [50]). Most importantly for the current study, macrophages release a spectrum of pyrimidine species of which deoxycytidine molecularly competes with gemcitabine uptake and metabolism thereby hindering its efficacy as a chemotherapy in PDAC [34]. We here confirm the importance of macrophage-derived deoxycytidine in gemcitabine resistance and identify $\mathrm{C} / \mathrm{EBP} \delta$ as a key transcription factor driving deoxycytidine biosynthesis in macrophages. In line with its specific effect on deoxycytidine biosynthesis, macrophage C /EBP $\delta$ did not impact resistance to either 5-FU or paclitaxel. Indeed, conditioned medium obtained from both wild type and C/EBP $\delta$-deficient macrophages did not induce resistance to 5-FU and paclitaxel, but actually slightly enhanced 5-FU- and paclitaxel-induced cytotoxicity in PANC-1 and BxPc3 cells. At this moment, we do not have an explanation for the small additive effect of macrophage conditioned medium on 5-FUand paclitaxel-induced cytotoxicity, but most importantly our data highlight that C/EBP specifically acts upon gemcitabine resistance of pancreatic cancer cells and that C/EBP $\delta$ is not a generic player in PDAC chemoresistance. Interestingly, however, pancreatic cancer cells are more resistant to gemcitabine than other chemotherapeutic drugs [46], making $\mathrm{C} / \mathrm{EBP} \delta$ an attractive transcription factor in the setting of chemoresistance in PDAC.

\section{Conclusions}

In conclusion, we here show that macrophage $\mathrm{C} / \mathrm{EBP} \delta$ drives gemcitabine resistance of pancreatic cancer cells in a deoxycytidine-dependent manner. Our data contribute to a better understanding of gemcitabine resistance in PDAC which may ultimately aid in improved prognosis of this dismal disorder.

Supplementary Materials: The following supporting information can be downloaded at: https: / / www.mdpi.com/article/10.3390/biomedicines10020219/s1, Figure S1: Dose-response curves of gemcitabine treatment, Figure S2: Conditioned medium does not affect cell viability, Figure S3: Dose-response curves of paclitaxel and 5-FU treatment, Table S1: Primers for LC validation.

Author Contributions: Conceptualization, C.A.S. and J.D.; methodology, C.A.S. and J.D.; validation, C.A.S. and J.D.; investigation, H.L.A. and J.D.; data curation, C.A.S., H.L.A. and J.D; writing-original draft preparation, C.A.S.; writing-review and editing, C.A.S. and J.D.; visualization, J.D. and C.A.S.; supervision, C.A.S. and J.D.; project administration, C.A.S. and J.D.; funding acquisition, J.D. All authors have read and agreed to the published version of the manuscript.

Funding: This research was supported by NWO (Netherlands Organization for Scientific Research), VENI grant (016.186.046). The APC was funded by the University of Amsterdam. The funders did not participate in the study design, data collection, data analysis, interpretation or writing of the report.

Institutional Review Board Statement: Not applicable.

Informed Consent Statement: Not applicable.

Data Availability Statement: The data presented in this study are available from the corresponding author upon reasonable request.

Conflicts of Interest: The authors declare no conflict of interest.

\section{References}

1. Ferlay, J.; Ervik, M.; Lam, F.; Colombet, M.; Mery, L.; Piñeros, M.; Znaor, A.; Soerjomataram, I.; Bray, F. Global Cancer Observatory: Cancer Today. Lyon: International Agency for Research on Cancer; 2020. Available online: https://gco.iarc.fr/today (accessed on 11 January 2022).

2. Rawla, P.; Sunkara, T.; Gaduputi, V. Epidemiology of Pancreatic Cancer: Global Trends, Etiology and Risk Factors. World J. Oncol. 2019, 10, 10-27. [CrossRef] [PubMed]

3. Siegel, R.L.; Miller, K.D.; Jemal, A. Cancer statistics, 2020. CA Cancer J. Clin. 2020, 70, 7-30. [CrossRef] [PubMed]

4. Kamisawa, T.; Wood, L.D.; Itoi, T.; Takaori, K. Pancreatic cancer. Lancet 2016, 388, 73-85. [CrossRef] 
5. $\quad$ Principe, D.R.; Underwood, P.W.; Korc, M.; Trevino, J.G.; Munshi, H.G.; Rana, A. The Current Treatment Paradigm for Pancreatic Ductal Adenocarcinoma and Barriers to Therapeutic Efficacy. Front. Oncol. 2021, 11, 688377. [CrossRef]

6. Von Hoff, D.D.; Ervin, T.; Arena, F.P.; Chiorean, E.G.; Infante, J.; Moore, M.; Seay, T.; Tjulandin, S.A.; Ma, W.W.; Saleh, M.N.; et al Increased Survival in Pancreatic Cancer with Nab-Paclitaxel Plus Gemcitabine. N. Engl. J. Med. 2013, 369, 1691-1703. [CrossRef]

7. Conroy, T.; Hammel, P.; Hebbar, M.; Ben Abdelghani, M.; Wei, A.C.; Raoul, J.L.; Choné, L.; Francois, E.; Artru, P.; Biagi, J.J.; et al. FOLFIRINOX or Gemcitabine as Adjuvant Therapy for Pancreatic Cancer. N. Engl. J. Med. 2018, 379, 2395-2406. [CrossRef]

8. Porta, C.; Riboldi, E.; Totaro, M.G.; Strauss, L.; Sica, A.; Mantovani, A. Macrophages in cancer and infectious diseases: The 'good' and the 'bad'. Immunotherapy 2011, 3, 1185-1202. [CrossRef]

9. Jadus, M.R.; Irwin, M.C.; Irwin, M.R.; Horansky, R.D.; Sekhon, S.; Pepper, K.A.; Kohn, D.B.; Wepsic, H.T. Macrophages can recognize and kill tumor cells bearing the membrane isoform of macrophage colony-stimulating factor. Blood 1996, 87, 5232-5241. [CrossRef]

10. Goswami, K.K.; Ghosh, T.; Ghosh, S.; Sarkar, M.; Bose, A.; Baral, R. Tumor promoting role of anti-tumor macrophages in tumor microenvironment. Cell Immunol. 2017, 316, 1-10. [CrossRef]

11. Jung, K.Y.; Cho, S.W.; Kim, Y.A.; Kim, D.; Oh, B.C.; Park, D.J.; Park, Y.J. Cancers with Higher Density of Tumor-Associated Macrophages Were Associated with Poor Survival Rates. J. Pathol. Transl. Med. 2015, 49, 318-324. [CrossRef]

12. Lindsten, T.; Hedbrant, A.; Ramberg, A.; Wijkander, J.; Solterbeck, A.; Eriksson, M.; Delbro, D.; Erlandsson, A. Effect of macrophages on breast cancer cell proliferation.; and on expression of hormone receptors.; uPAR and HER-2. Int. J. Oncol. 2017, 51, 104-114. [CrossRef]

13. Zhang, Q.W.; Liu, L.; Gong, C.Y.; Shi, H.S.; Zeng, Y.H.; Wang, X.Z.; Zhao, Y.W.; Wei, Y.Q. Prognostic significance of tumorassociated macrophages in solid tumor: A meta-analysis of the literature. PLoS ONE 2012, 7, e50946. [CrossRef] [PubMed]

14. Su, S.; Liu, Q.; Chen, J.; Chen, J.; Chen, F.; He, C.; Huang, D.; Wu, W.; Lin, L.; Huang, W.; et al. A positive feedback loop between mesenchymal-like cancer cells and macrophages is essential to breast cancer metastasis. Cancer Cell 2014, 25, 605-620. [CrossRef]

15. Fan, Q.M.; Jing, Y.Y.; Yu, G.F.; Kou, X.R.; Ye, F.; Gao, L.; Li, R.; Zhao, Q.D.; Yang, Y.; Lu, Z.H.; et al. Tumor-associated macrophages promote cancer stem cell-like properties via transforming growth factor-beta1-induced epithelial-mesenchymal transition in hepatocellular carcinoma. Cancer Lett. 2014, 352, 160-168. [CrossRef]

16. Liu, C.Y.; Xu, J.Y.; Shi, X.Y.; Huang, W.; Ruan, T.Y.; Xie, P.; Ding, J.L. M2-polarized tumor-associated macrophages promoted epithelial-mesenchymal transition in pancreatic cancer cells; partially through TLR4/IL-10 signaling pathway. Lab. Investig. 2013, 93, 844-854. [CrossRef]

17. Mitchem, J.B.; Brennan, D.J.; Knolhoff, B.L.; Belt, B.A.; Zhu, Y.; Sanford, D.E.; Belaygorod, L.; Carpenter, D.; Collins, L.; PiwnicaWorms, D.; et al. Targeting tumor-infiltrating macrophages decreases tumor-initiating cells.; relieves immunosuppression.; and improves chemotherapeutic responses. Cancer Res. 2013, 73, 1128-1141. [CrossRef]

18. Weizman, N.; Krelin, Y.; Shabtay-Orbach, A.; Amit, M.; Binenbaum, Y.; Wong, R.J.; Gil, Z. Macrophages mediate gemcitabine resistance of pancreatic adenocarcinoma by upregulating cytidine deaminase. Oncogene 2014, 33, 3812-3819. [CrossRef]

19. Adamska, A.; Domenichini, A.; Falasca, M. Pancreatic Ductal Adenocarcinoma: Current and Evolving Therapies. Int. J. Mol. Sci. 2017, 18, 1338. [CrossRef]

20. Buchholz, S.M.; Goetze, R.G.; Singh, S.K.; Ammer-Herrmenau, C.; Richards, F.M.; Jodrell, D.I.; Buchholz, M.; Michl, P.; Ellenrieder, V.; Hessmann, E.; et al. Depletion of Macrophages Improves Therapeutic Response to Gemcitabine in Murine Pancreas Cancer. Cancers 2020, 12, 1978. [CrossRef] [PubMed]

21. Lekstrom-Himes, J.; Xanthopoulos, K.G. Biological role of the CCAAT/enhancer-binding protein family of transcription factors. J. Biol. Chem. 1998, 273, 28545-28548. [CrossRef] [PubMed]

22. Gery, S.; Tanosaki, S.; Hofmann, W.K.; Koppel, A.; Koeffler, H.P. C/EBPdelta expression in a BCR-ABL-positive cell line induces growth arrest and myeloid differentiation. Oncogene 2005, 24, 1589-1597. [CrossRef]

23. Stein, T.; Salomonis, N.; Nuyten, D.S.; van de Vijver, M.J.; Gusterson, B.A. A mouse mammary gland involution mRNA signature identifies biological pathways potentially associated with breast cancer metastasis. J. Mammary Gland Biol. Neoplasia 2009, 14, 99-116. [CrossRef]

24. Ikezoe, T.; Gery, S.; Yin, D.; O’Kelly, J.; Binderup, L.; Lemp, N.; Taguchi, H.; Koeffler, H.P. CCAAT/enhancer-binding protein delta: A molecular target of 1.;25-dihydroxyvitamin D3 in androgen-responsive prostate cancer LNCaP cells. Cancer Res. 2005, 65, 4762-4768. [CrossRef] [PubMed]

25. Sivko, G.S.; Sanford, D.C.; Dearth, L.D.; Tang, D.; DeWille, J.W. CCAAT/Enhancer binding protein delta (c/EBPdelta) regulation and expression in human mammary epithelial cells: II. Analysis of activating signal transduction pathways; transcriptional; post-transcriptional; and post-translational control. J. Cell Biochem. 2004, 93, 844-856. [CrossRef] [PubMed]

26. Hartl, L.; Duitman, J.; Aberson, H.L.; Chen, K.; Dijk, F.; Roelofs, J.J.T.H.; Dings, M.P.G.; Hooijer, G.K.J.; Hernanda, P.Y.; Pan, Q.; et al. CCAAT/Enhancer-Binding Protein Delta (C/EBP8): A Previously Unrecognized Tumor Suppressor that Limits the Oncogenic Potential of Pancreatic Ductal Adenocarcinoma Cells. Cancers 2020, 12, 2546. [CrossRef] [PubMed]

27. Duitman, J.; Hartl, L.; Roelofs, J.J.T.H.; Bijlsma, M.F.; Spek, C.A. Non-Tumor CCAAT/Enhancer-Binding Protein Delta Potentiates Tumor Cell Extravasation and Pancreatic Cancer Metastasis Formation. Biomolecules 2021, 11, 1079. [CrossRef]

28. Chang, L.H.; Huang, H.S.; Wu, P.T.; Jou, I.M.; Pan, M.H.; Chang, W.C.; Wang, D.D.; Wang, J.M. Role of macrophage CCAAT/enhancer binding protein delta in the pathogenesis of rheumatoid arthritis in collagen-induced arthritic mice. PLoS ONE 2012, 7, e45378. [CrossRef] 
29. Banerjee, S.; Xie, N.; Cui, H.; Tan, Z.; Yang, S.; Icyuz, M.; Abraham, E.; Liu, G. MicroRNA let-7c regulates macrophage polarization. J. Immunol. 2013, 190, 6542-6549. [CrossRef]

30. Jones, V.S.; Huang, R.Y.; Chen, L.P.; Chen, Z.S.; Fu, L.; Huang, R.P. Cytokines in cancer drug resistance: Cues to new therapeutic strategies. Biochim. Biophys. Acta 2016, 1865, 255-265. [CrossRef]

31. Spek, C.A.; Aberson, H.L.; Butler, J.M.; de Vos, A.F.; Duitman, J. CEBPD Potentiates the Macrophage Inflammatory Response but CEBPD Knock-Out Macrophages Fail to Identify CEBPD-Dependent Pro-Inflammatory Transcriptional Programs. Cells 2021, 10, 2233. [CrossRef]

32. Slapak, E.J.; Kong, L.; El Mandili, M.; Nieuwland, R.; Kros, A.; Bijlsma, M.F.; Spek, C.A. ADAM9-Responsive Mesoporous Silica Nanoparticles for Targeted Drug Delivery in Pancreatic Cancer. Cancers 2021, 13, 3321. [CrossRef] [PubMed]

33. Halbrook, C.J.; Pontious, C.; Kovalenko, I.; Lapienyte, L.; Dreyer, S.; Lee, H.J.; Thurston, G.; Zhang, Y.; Lazarus, J.; Sajjakulnukit, P.; et al. Macrophage-Released Pyrimidines Inhibit Gemcitabine Therapy in Pancreatic Cancer. Cell Metab. 2019, 29, 1390-1399.e6. [CrossRef]

34. Shukla, S.K.; Purohit, V.; Mehla, K.; Gunda, V.; Chaika, N.V.; Vernucci, E.; King, R.J.; Abrego, J.; Goode, G.D.; Dasgupta, A.; et al. MUC1 and HIF-1alpha Signaling Crosstalk Induces Anabolic Glucose Metabolism to Impart Gemcitabine Resistance to Pancreatic Cancer. Cancer Cell 2017, 32, 71-87.e7. [CrossRef] [PubMed]

35. Amrutkar, M.; Gladhaug, I.P. Pancreatic Cancer Chemoresistance to Gemcitabine. Cancers 2017, 9, 157. [CrossRef] [PubMed]

36. Burris, H.A., 3rd; Moore, M.J.; Andersen, J.; Green, M.R.; Rothenberg, M.L.; Modiano, M.R.; Cripps, M.C.; Portenoy, R.K.; Storniolo, A.M.; Tarassoff, P.; et al. Improvements in survival and clinical benefit with gemcitabine as first-line therapy for patients with advanced pancreas cancer: A randomized trial. J. Clin. Oncol. 1997, 15, 2403-2413.

37. Cunningham, D.; Chau, I.; Stocken, D.D.; Valle, J.W.; Smith, D.; Steward, W.; Harper, P.G.; Dunn, J.; Tudur-Smith, C.; West, J.; et al. Phase III randomized comparison of gemcitabine versus gemcitabine plus capecitabine in patients with advanced pancreatic cancer. J. Clin. Oncol. 2009, 27, 5513-5518. [CrossRef]

38. Heinemann, V.; Quietzsch, D.; Gieseler, F.; Gonnermann, M.; Schönekäs, H.; Rost, A.; Neuhaus, H.; Haag, C.; Clemens, M.; Heinrich, B.; et al. Randomized phase III trial of gemcitabine plus cisplatin compared with gemcitabine alone in advanced pancreatic cancer. J. Clin. Oncol. 2006, 24, 3946-3952. [CrossRef]

39. Louvet, C.; Labianca, R.; Hammel, P.; Lledo, G.; Zampino, M.G.; André, T.; Zaniboni, A.; Ducreux, M.; Aitini, E.; Taïeb, J.; et al. Gemcitabine in combination with oxaliplatin compared with gemcitabine alone in locally advanced or metastatic pancreatic cancer: Results of a GERCOR and GISCAD phase III trial. J. Clin. Oncol. 2005, 23, 3509-3516. [CrossRef]

40. Conroy, T.; Desseigne, F.; Ychou, M.; Bouché, O.; Guimbaud, R.; Bécouarn, Y.; Adenis, A.; Raoul, J.L.; Gourgou-Bourgade, S.; de la Fouchardière, C.; et al. FOLFIRINOX versus gemcitabine for metastatic pancreatic cancer. N. Engl. J. Med. 2011, 364, 1817-1825. [CrossRef]

41. de Jesus, V.H.F.; Camandaroba, M.P.G.; Donadio, M.D.S.; Cabral, A.; Muniz, T.P.; de Moura Leite, L.; Sant'Ana, L.F. Retrospective comparison of the efficacy and the toxicity of standard and modified FOLFIRINOX regimens in patients with metastatic pancreatic adenocarcinoma. J. Gastrointest. Oncol. 2018, 9, 694-707. [CrossRef]

42. Noy, R.; Pollard, J.W. Tumor-associated macrophages: From mechanisms to therapy. Immunity 2014, 41, 49-61. [CrossRef] [PubMed]

43. Clappaert, E.J.; Murgaski, A.; Van Damme, H.; Kiss, M.; Laoui, D. Diamonds in the Rough: Harnessing Tumor-Associated Myeloid Cells for Cancer Therapy. Front. Immunol. 2018, 9, 2250. [CrossRef] [PubMed]

44. Ullmann, T.; Luckhardt, S.; Wolf, M.; Parnham, M.J.; Resch, E. High-Throughput Screening for CEBPD-Modulating Compounds in THP-1-Derived Reporter Macrophages Identifies Anti-Inflammatory HDAC and BET Inhibitors. Int. J. Mol. Sci. 2021, $22,3022$. [CrossRef] [PubMed]

45. Chi, J.Y.; Hsiao, Y.W.; Li, C.F.; Lo, Y.C.; Lin, Z.Y.; Hong, J.Y.; Liu, Y.M.; Han, X.; Wang, S.M.; Chen, B.K.; et al. Targeting chemotherapy-induced PTX3 in tumor stroma to prevent the progression of drug-resistant cancers. Oncotarget 2015, 6, 2398724001. [CrossRef]

46. Zeng, S.; Pöttler, M.; Lan, B.; Grützmann, R.; Pilarsky, C.; Yang, H. Chemoresistance in Pancreatic Cancer. Int. J. Mol. Sci. 2019, 20, 4504. [CrossRef]

47. Schniewind, B.; Christgen, M.; Kurdow, R.; Haye, S.; Kremer, B.; Kalthoff, H.; Ungefroren, H. Resistance of pancreatic cancer to gemcitabine treatment is dependent on mitochondria-mediated apoptosis. Int. J. Cancer 2004, 109, 182-188. [CrossRef]

48. Fu, Y.; Ricciardiello, F.; Yang, G.; Qiu, J.; Huang, H.; Xiao, J.; Cao, Z.; Zhao, F.; Liu, Y.; Luo, W.; et al. The Role of Mitochondria in the Chemoresistance of Pancreatic Cancer Cells. Cells 2021, 10, 497. [CrossRef]

49. Rauchwerger, D.R.; Firby, P.S.; Hedley, D.W.; Moore, M.J. Equilibrative-sensitive nucleoside transporter and its role in gemcitabine sensitivity. Cancer Res. 2000, 60, 6075-6079.

50. Yang, S.; Liu, Q.; Liao, Q. Tumor-Associated Macrophages in Pancreatic Ductal Adenocarcinoma: Origin, Polarization, Function, and Reprogramming. Front. Cell Dev. Biol. 2021, 8, 607209. [CrossRef] 